\title{
Harnessing alveolar macrophages for sustained mucosal T-cell recall confers long-term protection to mice against lethal influenza challenge without clinical disease
}

\author{
DC Macdonald ${ }^{1}$, H Singh ${ }^{1}$, MA Whelan ${ }^{2}$, D Escors ${ }^{1}$, F Arce ${ }^{1}$, SE Bottoms ${ }^{2}$, WS Barclay ${ }^{3}$, M Maini $^{1}$, \\ MK Collins ${ }^{1,4}$ and WMC Rosenberg ${ }^{2,4}$
}

Vaccines that induce T cells, which recognize conserved viral proteins, could confer universal protection against seasonal and pandemic influenza strains. An effective vaccine should generate sufficient mucosal T cells to ensure rapid viral control before clinical disease. However, T cells may also cause lung injury in influenza, so this approach carries inherent risks. Here we describe intranasal immunization of mice with a lentiviral vector expressing influenza nucleoprotein (NP), together with an NFאB activator, which transduces over $75 \%$ of alveolar macrophages (AM). This strategy recalls and expands NP-specific CD8 + T cells in the lung and airway of mice that have been immunized subcutaneously, or previously exposed to influenza. Granzyme B-high, lung-resident T-cell populations persist for at least 4 months and can control a lethal influenza challenge without harmful cytokine responses, weight loss, or lung injury. These data demonstrate that AM can be harnessed as effective antigen-presenting cells for influenza vaccination.

\section{INTRODUCTION}

Over three decades ago, it was established that $\mathrm{T}$ cells have an essential role in influenza clearance in mouse models. ${ }^{1}$ Adoptive transfer of influenza-specific CD8 $+\mathrm{T}$ cells shortens infection, ${ }^{2}$ whereas depletion of the same subset leads to more severe disease and reduced survival. ${ }^{3}$ Importantly, memory $\mathrm{T}$ cells generated by infection can confer partial protection against other influenza strains (heterosubtypic immunity). ${ }^{4}$ Generation of T-cell memory through immunization can limit duration or severity of influenza in animals (for example, Donnelly et al. ${ }^{5}$ ). In humans, viral shedding is reduced in the presence of cytotoxic T-cell memory from previous infection, despite the absence of specific antibodies. ${ }^{6}$ As the T-cell epitopes of virion proteins such as nucleoprotein (NP) and matrix protein 1 (M1) are highly conserved, vaccines expressing these antigens have the potential to confer cross-protection against seasonal and emergent pandemic strains. ${ }^{7}$ Promising initial findings from a clinical study immunizing volunteers intramuscularly with modified vaccinia virus Ankara encoding $\mathrm{NP}+\mathrm{M} 1$ have demonstrated a reduction, though not elimination, of symptoms following influenza challenge. ${ }^{8}$

With the arrival of tetramer technology, it was shown that much larger numbers of influenza-specific CD8 T-cell populations are generated in murine lung and airway after primary infection than previously estimated. ${ }^{9}$ These have a predominant effector phenotype and appear to be replenished from circulating effectors for several weeks after infection rather than a self-sustaining established memory population. ${ }^{10}$ Importantly, equivalent numbers of lung-based influenzaspecific $\mathrm{T}$ cells are not induced in mice infected intraperitoneally or vaccinated by non-pulmonary routes. In these circumstances, localization of memory $\mathrm{T}$ cells to the lung is delayed up to 5 days after infection, and correspondingly, control of heterosubtypic infection is greatly attenuated. ${ }^{11}$ Conversely, after pulmonary infection, spleen or mediastinal lymph nodes can be removed without detriment to the size,

\footnotetext{
${ }^{1}$ Division of Infection and Immunity and MRC Centre for Medical Molecular Virology, University College London, London, UK. ${ }^{2}$ Division of Medicine, University College London, London, UK and ${ }^{3}$ Division of Virology, Imperial College London, St Mary's Campus, London, UK. Correspondence: MK Collins (mary.collins@ucl.ac.uk)

${ }^{4}$ These authors contributed equally to this work.
} 
speed, or efficacy of the T-cell response to a subsequent heterosubtypic challenge. ${ }^{12,13}$ Therefore, a mucosal rather than systemic T-cell vaccine approach may be more successful at limiting, or even preventing, clinical disease after influenza infection.

However, immunoactivation in the lung is tightly controlled, protecting its large and delicate surface area which is continually exposed to foreign antigen and readily disrupted by inflammation. This presents a considerable barrier to T-cell priming and local memory maintenance. ${ }^{14}$ Also, numbers of lung memory $\mathrm{T}$ cells start to wane some 15 weeks after primary infection in mice, correlating with declining immunity to heterosubtypic challenge. ${ }^{3}$ We endeavoured to circumvent the high barrier to mucosal T-cell immunoactivation by establishing lung-based $\mathrm{T}$-cell populations by recall rather than by direct mucosal priming. Lentiviral vectors (LV), based on HIV-1, are replication defective, do not encode any HIV proteins, and can transduce non-dividing cells, including primary human dendritic cells and macrophages. ${ }^{15} \mathrm{LV}$ have been reported to transduce murine alveolar macrophages (AM) after intranasal (IN) delivery, ${ }^{16}$ and although the T-cell priming potential of AM is limited, ${ }^{17}$ the potential of harnessing these cells for recall has not been previously explored.

Here we show that IN immunization with a LV encoding NP together with an NFKB activator ${ }^{18}$ transduces AM with high efficiency, resulting in potent T-cell recruitment by chemoattractant secretion and antigen-driven expansion. In mice that have been primed by subcutaneous (SC) immunization or previous influenza infection, this results in substantial NPspecific T-cell populations in the lung and airway, conferring long-lasting cross-strain protection against lethal influenza challenge without associated lung injury or weight loss.

\section{RESULTS}

\section{LV can efficiently transduce and activate AM after IN administration}

We constructed LV expressing influenza A/PR/8/34 H1N1 (PR8) NP (or the marker protein green fluorescent protein (GFP)) together with a constitutive activator of the NFkB pathway, Kaposi's sarcoma-associated herpesvirus FLICE-like inhibitory protein (vFLIP; ${ }^{18,19}$ or a non-coding insert: Null;
Figure 1). LV with the ubiquitin promoter (UBI) were used in the majority of experiments, while the phosphoglycerate kinase promoter was used in human cells (Figure 7C). We included vFLIP because influenza infection of $\mathrm{AM}$ is known to drive NFkB-dependent secretion of non-ELR CXC (C-X-C motif chemokine receptor) chemokines, which preferentially attract mononuclear cells. ${ }^{20}$ Among these, IP-10 (interferon-inducible protein of $10 \mathrm{kd}$ ), MIP- $1 \alpha$ (macrophage inflammatory protein $1 \alpha$ ), MIP-1 $\beta$, MCP-1 (monocyte chemotactic protein-1), MCP-3, and RANTES (regulated upon activation, normal $\mathrm{T}$-cell expressed and secreted) are essential for T-cell recruitment in response to influenza. ${ }^{21}$

LV expressing vFLIP and GFP (vFLIP-GFP), or GFP alone (Null-GFP), or live PR8 influenza were administered IN to naive $\mathrm{BALB} / \mathrm{c}$ mice. Analysis of bronchoalveolar lavage (BAL) cells 4 days later revealed efficient $\mathrm{LV}$ transduction (>75\%) of $\mathrm{F} 4 / 80^{\mathrm{HI}} \mathrm{CD} 11 \mathrm{c}^{\mathrm{HI}}$ AM (Figure 2a histogram) with very high specificity ( $>99 \%$, adjacent fluorescence-activated cell sorted (FACS) plots). Intranasal administration of vFLIP-GFP attracted further macrophages and dendritic cells to the BAL (Figure 2a) and resulted in greatly increased levels of T-cell chemokines compared with Null-GFP recipients or saline controls (Figure 2b). When BAL cells were cultured for 4 days, high levels of T-cell chemokines were found in the supernatants of adherent cells (which were $\mathrm{F} 4 / 80^{\mathrm{HI}} \mathrm{CD} 11 \mathrm{c}^{\mathrm{HI}}$ after culture) from the vFLIP-GFP mice, demonstrating that AMs are the source of chemokines (Figure 2b).

\section{SC immunization with vFLIP-NP generates enhanced T-cell responses}

We have previously shown that LV co-expressing vFLIP enhance CD8 + T-cell responses to a model antigen (ovalbumin) in BL/6 mice. ${ }^{18}$ Here we confirm that a LV encoding vFLIP was as effective as lipopolysaccharide (LPS) in balb/c dendritic cell (DC) activation in vitro, significantly upregulating co-stimulatory molecules, PDL-1 and MHC (major histocompatibility complex) II expression (Supplementary Figure S1A). SC vaccination with vFLIP-NP generated greater interferon $\gamma($ IFN $\gamma)$ and GzmB CD8 + T-cell responses than Null-NP upon re-stimulation of splenocytes at day 14 (Supplementary Figure S1B) and un-stimulated ex vivo analysis of $\mathrm{NP}_{147-155}$ pentamer revealed higher GzmB and a

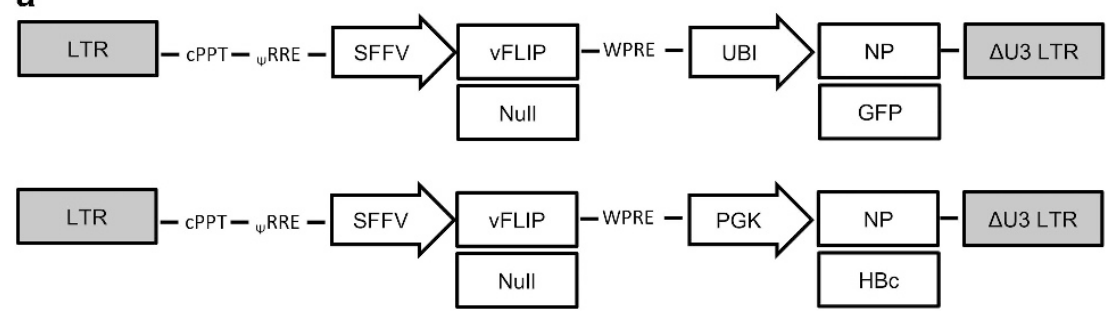

b

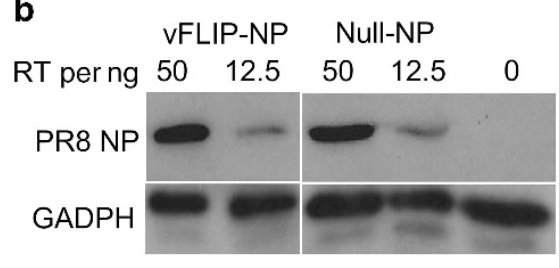

Figure 1 Lentiviral vector construction and testing. (a) Lentiviral vectors LV used in mouse (top) and human (bottom) experiments. vFLIP (Kaposi's sarcoma-associated herpesvirus FLICE-like inhibitory protein) is driven from the spleen focus forming virus (SFFV) promoter. "Null" indicates a noncoding 6 base-pair sequence. A/PR/8/34 H1N1 (PR8) nucleoprotein (NP) or GFP are driven from a ubiquitin (UBI) promoter in mouse experiments and a phosphoglycerate kinase (PGK) promoter in human cell experiments. (b) Immunoblot showing NP expression in 293T cells transduced with the dose of LV indicated. CPPT, central polypurine tract; GAPDH, glyceraldehyde 3-phosphate dehydrogenase; HBc, hepatitis B core; LTR, long terminal repeat; RRE, Rev-responsive element; RT, reverse transcriptase; WPRE, wood chuck hepatitis virus posttranscriptional regulatory element. 
a

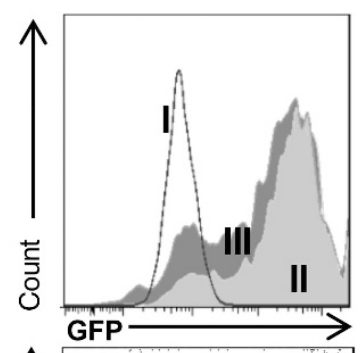

$\uparrow$ Saline

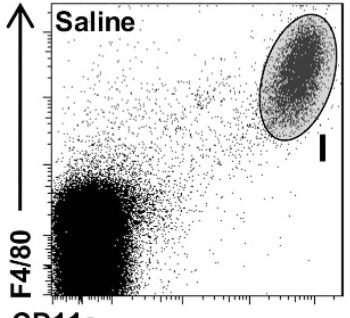

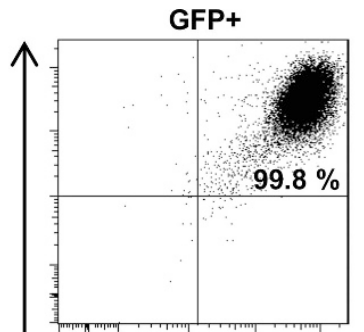

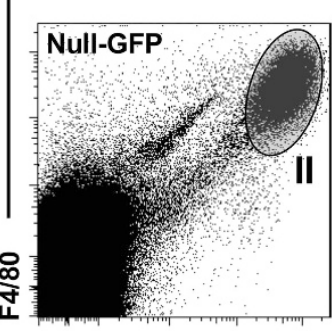

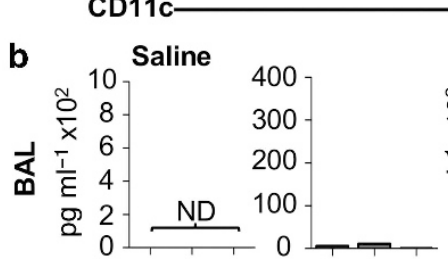
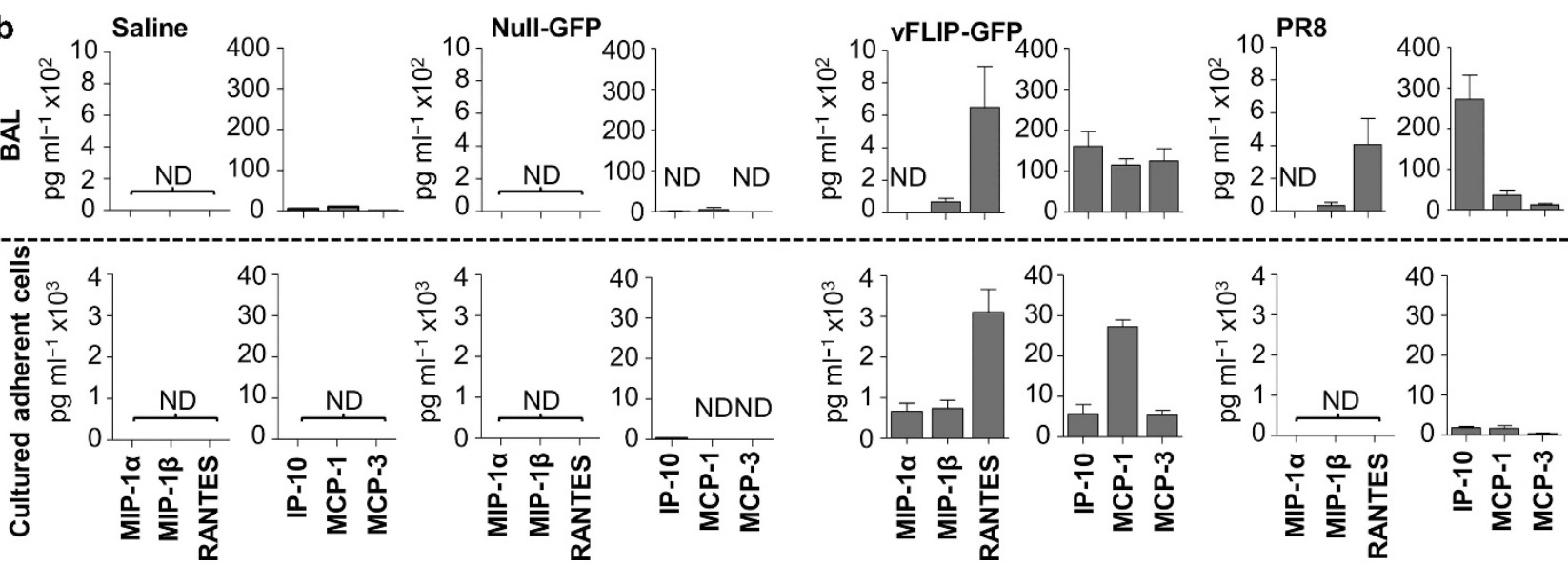

Figure 2 vFLIP (Kaposi's sarcoma-associated herpesvirus FLICE-like inhibitory protein)-transduced alveolar macrophages (AM) produce T-cell chemo-attractants. (a) AMare transduced by lentiviral vectors (LV) with high efficiency and specificity. In all, $200 \mathrm{ng}$ (RT) of LV encoding vFLIP-GFP (green fluorescent protein) or Null-GFP ( $n=3$ per group) were introduced intranasally. Bronchoalveolar lavage (BAL) was performed 4 days later and retrieved cells were stained for $\mathrm{F} 4 / 80$ and $\mathrm{CD} 11 \mathrm{c}$ and analyzed by fluorescence-activated cell sorter (FACS) (bottom panel). BAL cells from mice Day 4 after infection with influenza A/PR/8/34 are shown for comparison. Circled areas indicate F4/80 ${ }^{\mathrm{Hl}} \mathrm{CD} 11 \mathrm{c}^{\mathrm{HI}}$ AM populations in the naive, Null-GFP, and vFLIP-GFP groups. Histogram indicates transduction efficiency $(>75 \%)$ as indicated by GFP signal in AM (F4/80 $\left.{ }^{\mathrm{HI}} \mathrm{CD} 11 \mathrm{c}^{\mathrm{HI}}\right)$ populations. Adjacent FACS plots (top) indicate LV transduce only $\mathrm{F} 4 / 80^{\mathrm{HI}} \mathrm{CD} 11 \mathrm{c}^{\mathrm{HI}}$ cells. (b) vFLIP-transduced AM produce quantities of T-cell chemo-attractants comparable with mice infected with influenza A/PR/8/34. Concentrations of MIP-1 $\alpha$ (macrophage inflammatory protein $1 \alpha$ ), MIP-1 $\beta$, RANTES (regulated upon activation, normal T cell expressed and secreted), IP-10 (interferon-inducible protein of $10 \mathrm{kd}$ ), MCP-1 (monocyte chemotactic protein-1), and MCP-3 were measured in a single $2 \mathrm{ml} \mathrm{BAL} \mathrm{(} n=5$, top row) at Day 4 after intranasal LV or influenza infection. These were also measured in $2 \mathrm{ml}$ supernatants of adherent cells from BAL after 4 days culture in vitro (bottom row). FACS analysis of cultured adherent cells showed $>97 \%$ of transduced cells (GFP positive) were $\mathrm{F} 4 / 80^{\mathrm{HI}} \mathrm{CD} 11 \mathrm{c}^{\mathrm{HI}}$. Transduced (GFP +) CD11cHI F4/80 - DCs were not detected by FACS before or after culture of BAL cells. ND, not detected; RT, reverse transcriptase.

Ki67 expression in the vFLIP-NP group vs. Null-NP (Supplementary Figure S1C). CD4 + T-cell cytokine responses to re-stimulation with the class II-restricted $\mathrm{NP}_{57-78}$ peptide were also increased (Supplementary Figure S1D,E). Antibody responses against NP could not be detected following vFLIP-NP or Null-NP SC vaccination (data not shown), which may be attributed to endogenous antigen expression by this non-cytopathic vector and thus minimal B-cell exposure.

\section{Intranasal boosting with vFLIP-NP generates airway CD8 $+T$ cells and protects against influenza}

We next compared the ability of SC and IN immunization with vFLIP-NP LV to generate populations of antigen-specific
$\mathrm{CD} 8+\mathrm{T}$ cells in the lung. SC administration of either vFLIP$\mathrm{NP}$ or Null-NP resulted in a frequency of $\mathrm{NP}_{147-155}$ pentamer + positive CD8 + T cells in exsanguinated lung comparable with that found in the circulation or spleen (percentage of total $\mathrm{CD} 8+\mathrm{T}$ cells that are $\mathrm{NP}_{147-155}$ pentamer + after vFLIP-NP: lung $4.2 \pm 0.64 \%$, circulation $5.8 \pm 1.9 \%$, spleen $6 \pm 0.9 \%$; NullNP: lung $2.4 \pm 3.2$, circulation: $4.5 \pm 1.5 \%$, spleen $4.8 \pm 0.6 \%$ ). Two SC immunizations with vFLIP-NP resulted in $80 \%$ survival following lethal PR8 influenza challenge, but all mice lost considerable weight (15\% on average, Figure 3a). Administration of IN vFLIP-NP or Null-NP to naive mice failed to generate detectable CD8 $+\mathrm{NP}_{147-155}$ pentamer + $\mathrm{T}$ cells in airway, lung homogenate, circulation, or spleen. 
a
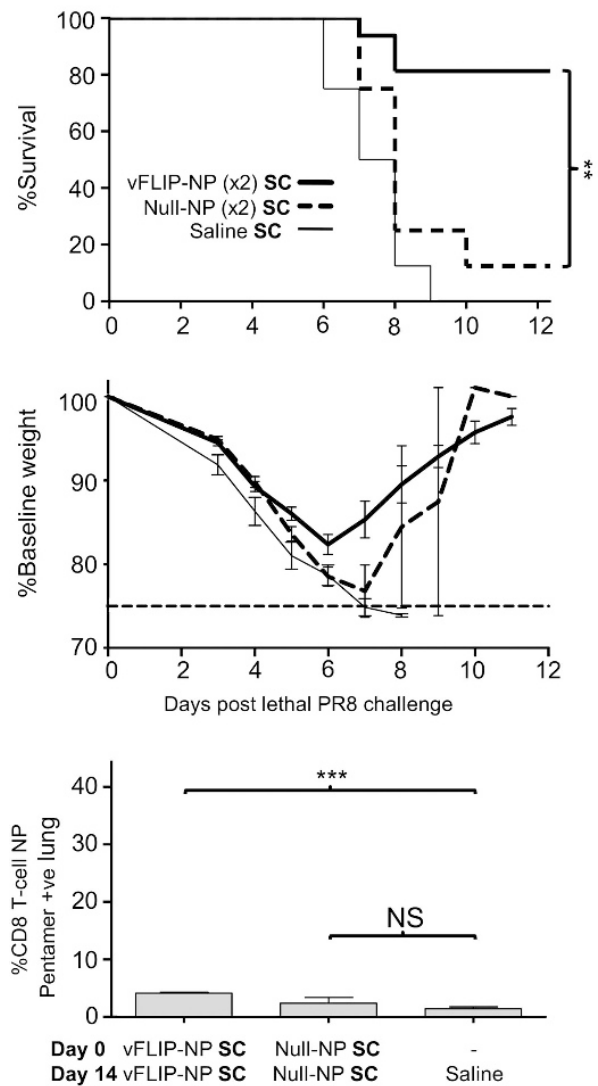

b
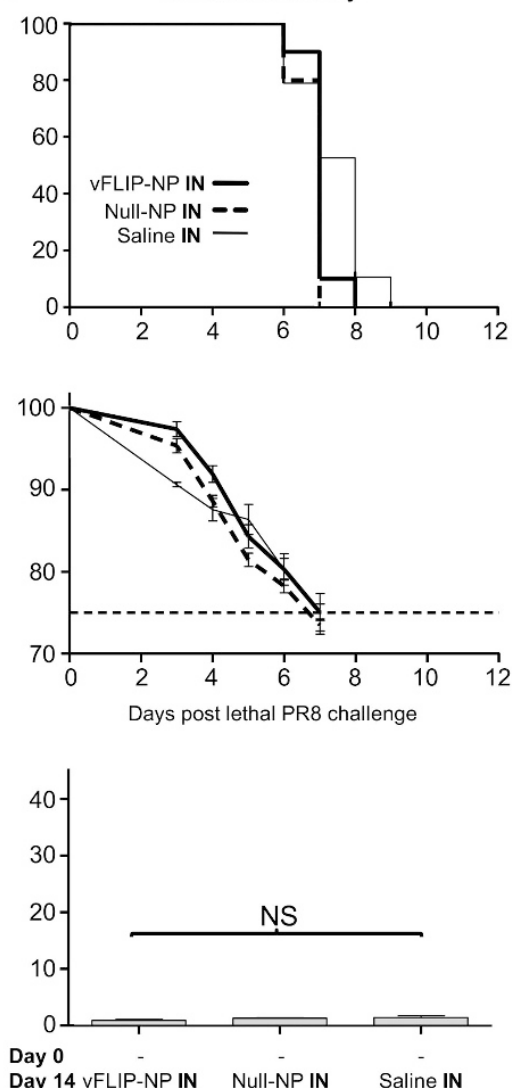

c
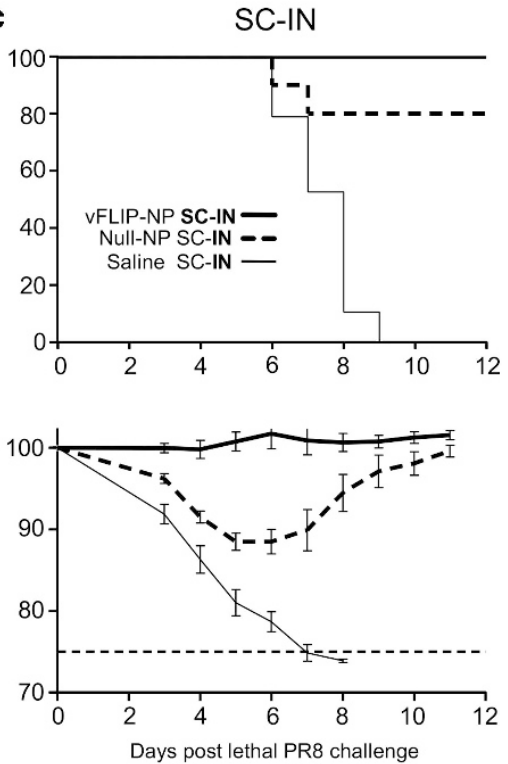

$\star *$

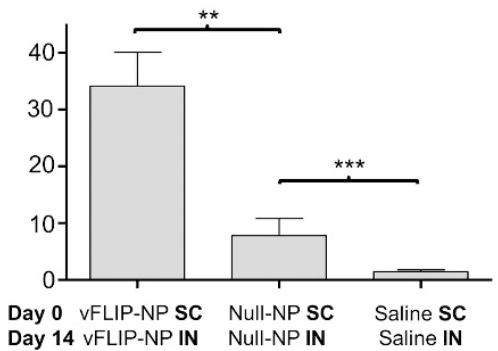

Figure 3 Response to (a) subcutaneous (SC), (b) intranasal (IN), or (c) SC followed by IN (SC-IN) immunization. Mice were challenged with lethal A/PR/8/34 (PR8) or killed for analysis 2 weeks after the final immunization of the regimen indicated on the $x$ axis. Top: Survival after lethal $2 \times$ LD $_{50}$ challenge ( $n=10-18$ per group). Both Mantel-Cox tests and Gehan-Breslow-Wilcoxon test produced similar $P$-values for observed differences in survival. Middle: Weight loss from baseline after lethal PR8 challenge $(n=10-18$ per group). Average weights are taken from all survivors in the group at that time point. Bottom: Percentage of $\mathrm{NP}_{147-155}$ pentamer-positive CD8 T cells from lung homogenate $(n=4-6$ per group). Mean values were compared by two-tailed Student's $t$-test. Error bars indicate s.e. of the mean. NP, nucleoprotein; NS, not significant; vFLIP, Kaposi's sarcoma-associated herpesvirus FLICE-like inhibitory protein.

Correspondingly, there was no detectable protection from lethal influenza challenge (Figure 3b). However, mice primed with vFLIP-NP SC and then boosted with vFLIP-NP IN (SC-IN, Figure 3c) accumulated very large proportions of $\mathrm{NP}_{147-155}$-specific CD8 $+\mathrm{T}$ cells in lung homogenate in excess of one-third of the total. Strikingly, this conferred complete protection against lethal PR8 challenge without any weight loss (Figure 3c).

Numbers of antigen-specific CD8 + T cells in mouse airway are known to correlate with the degree of protection against secondary influenza infection. ${ }^{3}$ Mice that received vFLIP-NP SC-IN immunization had $>60$-fold more CD8 $+\mathrm{T}$ cells in BAL at day 10 than unimmunized mice and twice more than those receiving Null-NP SC-IN (Figure 4a). CD8 + T cells were high in GzmB and Ki67, demonstrating cytotoxic potential and on-going proliferation (Figure 4a). Proportions of GzmB-positive $\mathrm{NP}_{147-155}$ pentamer CD8 $+\mathrm{T}$ cells were significantly greater in the vFLIP NP SC-IN group than in the Null-NP SC-IN group ( $51 \%$ vs. $26 \%, P=0.0031)$ (Figure 4a).
The enhanced T-cell response in the presence of vFLIP was selective for CD8 + cells. Both Null-NP and vFLIP-NP stimulated accumulation of Ki67 $+\mathrm{CD} 4+\mathrm{T}$ cells in BAL (Figure 4a). Cytokine responses of re-stimulated total lung $\mathrm{CD} 8+$ and $\mathrm{CD} 4+\mathrm{T}$ cells were minimal. Re-stimulation with either MHC I- or II-restricted NP peptides generated low percentages of IFN $\gamma$, tumor necrosis factor $\alpha$ (TNF $\alpha$ ), and interleukin (IL)-2-positive CD8 + and $\mathrm{CD} 4+\mathrm{T}$ cells and no significant differences between the vFLIP-NP and Null-NP SC-IN immunized groups (Supplementary Figure S2).

\section{Both vFLIP and NP are required to maximize T-cell recall and antigen-specific expansion}

We postulated that the major components of a recall response-chemotactic recruitment of memory cells and antigen-driven expansion-are principally instigated by transduction of AM with the components vFLIP and NP, respectively, and therefore numbers of antigen-specific $\mathrm{T}$ cells would be diminished in either's absence. To test this, we gave an IN LV expressing either vFLIP (vFLIP-GFP) or NP (Null-NP) 

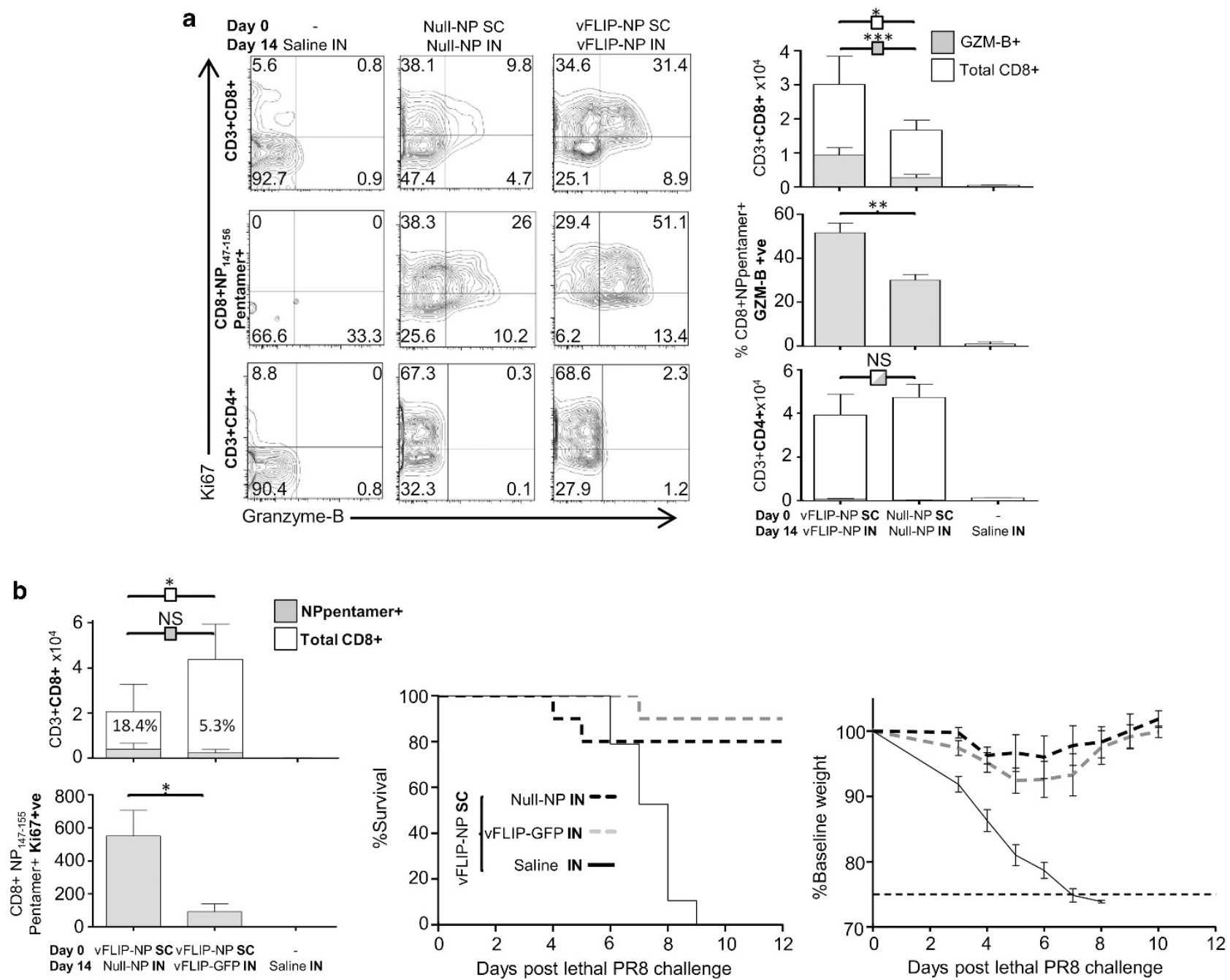

Figure 4 Analysis of airway T cells following SC-IN (subcutaneous followed by intranasal) immunization. (a) Fluorescence-activated cell sorted plots showing Granzyme B (GzmB) and Ki67 expression of total CD8 + T cells, nucleoprotein (NP)-pentamer positive CD8 + T cells, and total CD4 + T cells. Histograms show quantitative comparisons in these populations between groups of mice $(n=5)$ SC-IN immunized with vFLIP (Kaposi's sarcomaassociated herpesvirus FLICE-like inhibitory protein)-NP compared with Null-NP. (b) Comparison of airway T-cell populations (left), survival (middle), and weight loss (right) after SC vFLIP-NP immunization followed by intranasal vFLIP-GFP (green fluorescent protein) or Null-NP. NS, not significant.

to mice that had been immunized with vFLIP-NP SC 2 weeks earlier. The vFLIP-GFP IN recall increased total numbers of airway CD8 $\mathrm{T}$ cells (Figure $\mathbf{4 b}$, left), but the proportion of antigen-specific NP-positive CD8 T cells remained similar to that seen in the circulation and spleen. By contrast, Null-NP IN recall recruited less than half the number of total CD8 $+\mathrm{T}$ cells to the airway as vFLIP-GFP, but a greater proportion of these were NP-pentamer positive $(18.4 \%$ vs. $5.2 \%, P=0.002)$. This is consistent with local antigen-driven proliferation of $\mathrm{NP}$-specific CD8 $+\mathrm{T}$ cells, corroborated by the finding of sixfold higher numbers of Ki67 $+\mathrm{CD} 8+\mathrm{NP}_{147-155}$ pentamer $+\mathrm{T}$ cells in the airways of Null-NP IN recipients vs. the vFLIP-GFP IN recall group (Figure $4 b$ ). Both vFLIPGFP and Null-NP IN immunizations after vFLIP-NP SC priming generated similar absolute numbers of antigen-specific $\mathrm{T}$ cells in the airways but approximately threefold lower than that seen with vFLIP-NP SC-IN recall. Correspondingly, survival and weight loss after lethal influenza challenge were inferior to the complete protection observed with vFLIP-NP SC-IN immunization (Figure $4 \mathbf{b}$, middle and right).

\section{Adoptive transfer of transduced AM elicits lung T-cell recall and protection against influenza}

Although LV transduce AM with very high specificity among airway immune cells, they have also been reported to transduce approximately $10 \%$ of alveolar epithelial cells. ${ }^{16}$ To determine whether AM alone are necessary to instigate T-cell recall, we attempted their depletion by clodronate liposomes ${ }^{22}$ or diphtheria toxin in CD11c-DTR transgenic mice ${ }^{23}$ before IN LV vaccination. However, both approaches resulted in only partial AM depletion (clodronate liposomes mean 58\% reduction in AM compared with phosphate-buffered saline liposomes; CD11c-DTR transgenic model mean $60 \%$ reduction in AM with diphtheria toxin compared with saline). We 


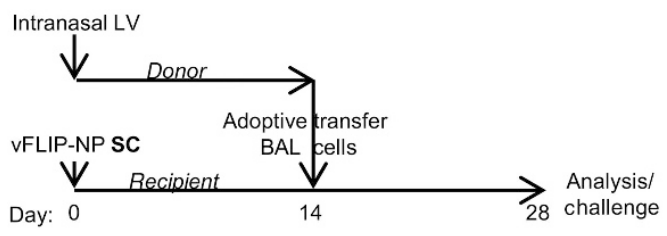

b

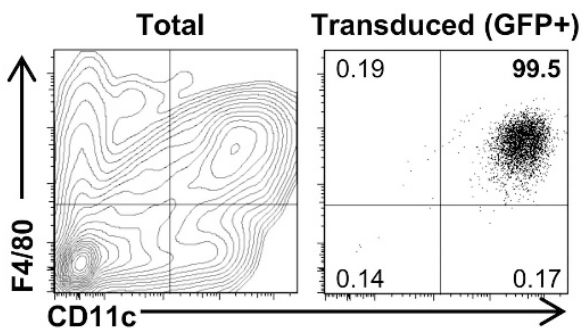

c

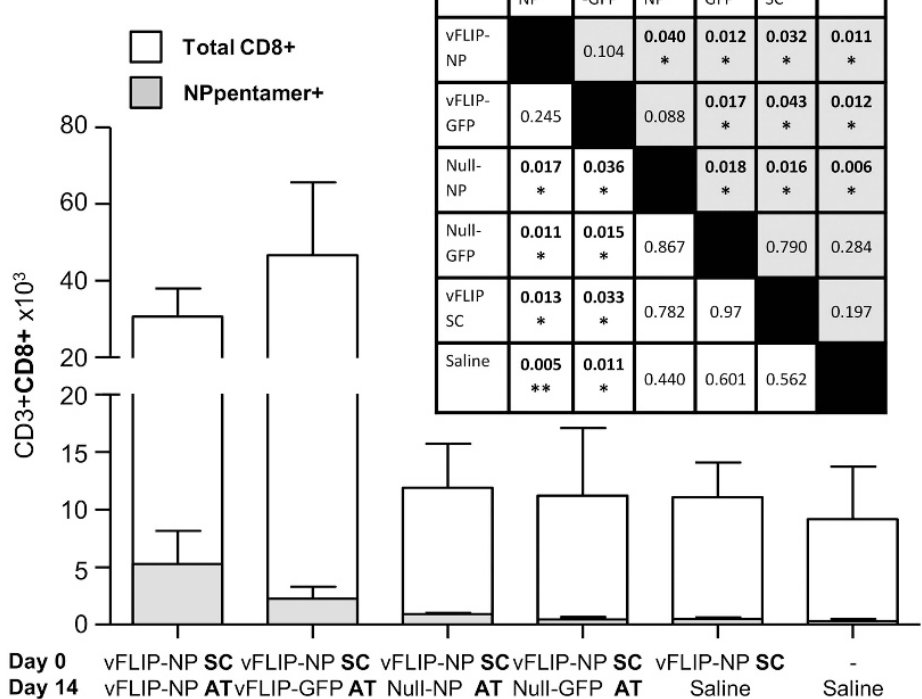

d

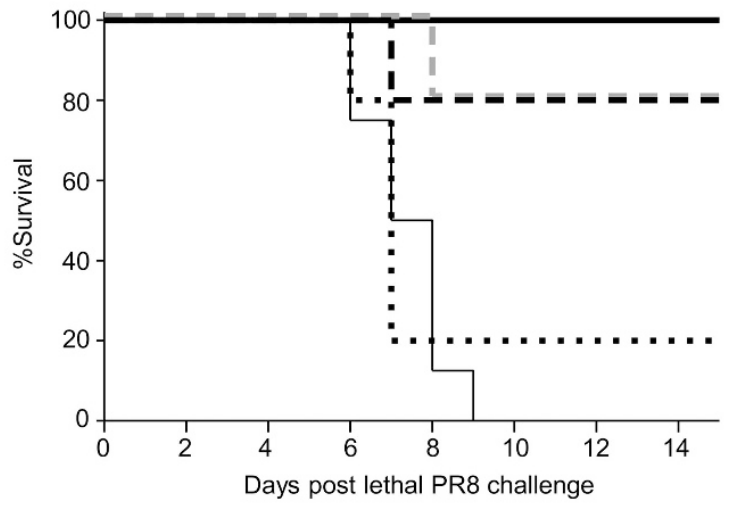

e

$$
\begin{aligned}
& \text { vFLIP-NP AT - } \\
& \text { Null-NP AT - } \\
& \text { vFLIP-GFP AT - } \\
& \text { Null-GFP AT --- } \\
& \text { Saline SC }
\end{aligned}
$$

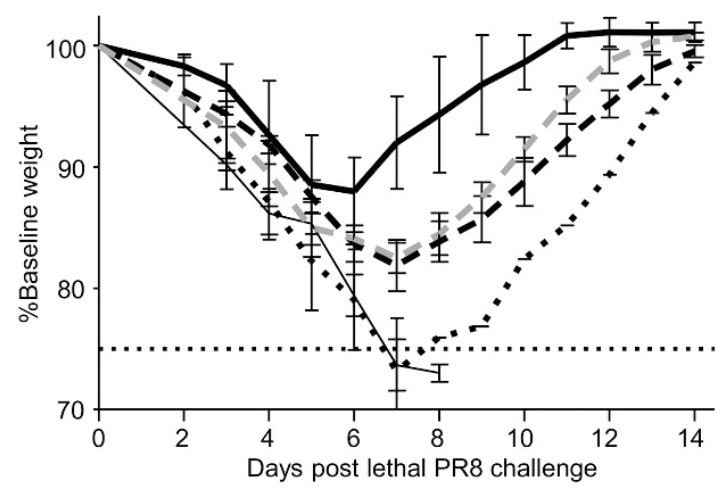

Figure 5 Adoptive transfer (AT) of lentiviral vector (LV)-transduced alveolar macrophages (AM) recall large lung T-cell populations and protects against lethal influenza challenge. (a) Adoptive transfer schedule. (b) Bronchoalveolar lavage (BAL) cells from donor mice were washed and concentrated at $12,000 \mathrm{AM}(\mathrm{F} / 480+\mathrm{CD} 11 \mathrm{c}+)$ cells per recipient mouse $(40 \mu \mathrm{l})$. Fluorescence-activated cell sorted plots show high specificity of $\mathrm{AM}(\mathrm{F} / 480+\mathrm{CD} 11 \mathrm{c}+$ ) transduction in donor cells. $\mathrm{NP}_{147-155}$ pentamer-positive CD8 $+\mathrm{T}$ cells were undetectable in BAL from donor mice (as in Figure 4b). (c) CD8 + T-cell populations from total lung homogenate of recipient mice 2 weeks after adoptive transfer. Comparative $T$-test $P$-values are given in the inset table. (d) Survival and (e) weight loss in survivors of recipient mice challenged 2 weeks after adoptive transfer. GFP, green fluorescent protein; NP, nucleoprotein; SC, subcutaneous; vFLIP, Kaposi's sarcoma-associated herpesvirus FLICE-like inhibitory protein.

therefore adoptively transferred AM transduced in vivo with IN LV into recipient mice that had been primed with SC vFLIP-NP 2 weeks previously (Figure 5a). This approach takes advantage of the $>99 \%$ specificity of AM transduction in the transferred population (Figure 5b), removing the need for cell sorting which incurs substantial processing losses of this highly adherent population. Two weeks after IN adoptive transfer, recipient mouse lungs were harvested from three mice per group for analysis of total lung $\mathrm{T}$ cells. This revealed a very similar pattern of T-cell recall as direct IN administration of LV (Figure 5c). Although absolute numbers of $\mathrm{NP}_{147-155}$ pentamer $+\mathrm{CD} 8+\mathrm{T}$ cells recalled by this means were threefold lower than achieved by direct IN LV, it is noteworthy that only $12,000 \mathrm{AM}$ were transferred, representing some $0.4 \%$ of the estimated total that would be transduced by direct IN LV administration. ${ }^{24}$ Despite this, adoptive transfer of vFLIP-NPtransduced donor AM induced a sufficient $\mathrm{T}$-cell recall response to confer $100 \%$ survival against lethal challenge with minimal weight loss (Figure 5d,e).

\section{Intranasal immunization limits influenza replication without lung injury or cytokine production}

$\mathrm{T}$ cells are known mediators of lung injury during influenza infection. Generating large populations of lung- and airwaybased T cells by vFLIP-NP SC-IN immunization thus has potential to enhance tissue injury upon influenza infection. We therefore examined the relationship between T-cell number, secondary effector cytokine responses and viral load after influenza infection in mice immunized with vFLIP-NP by SC or SC-IN regimens (Figure 6). In the SC-IN group effector 
a

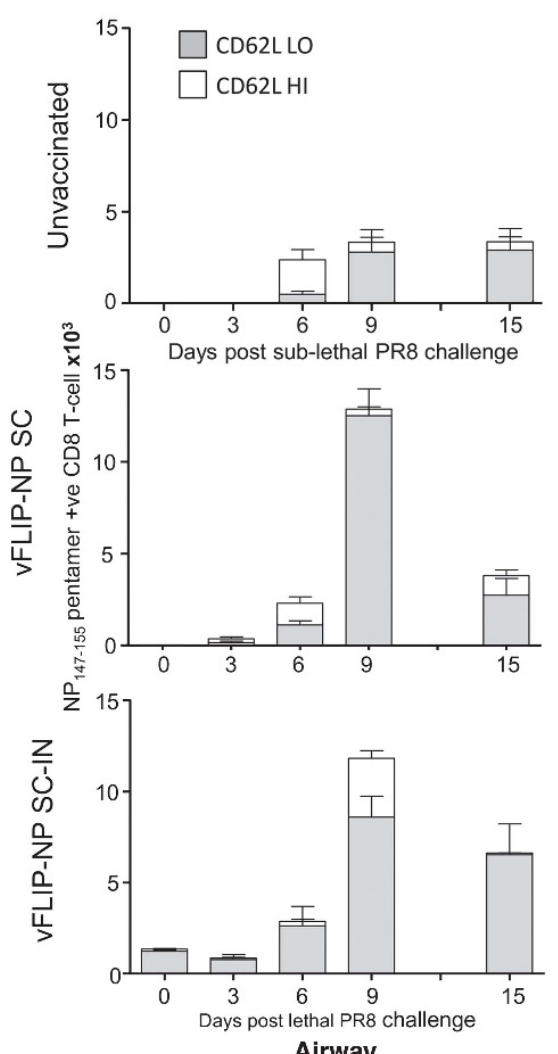

b

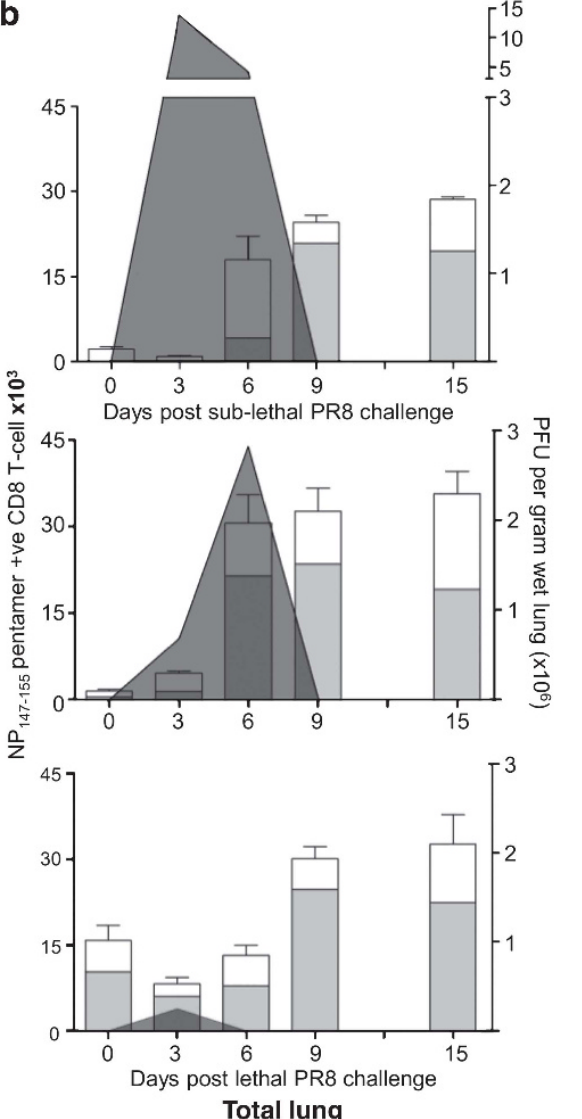

C

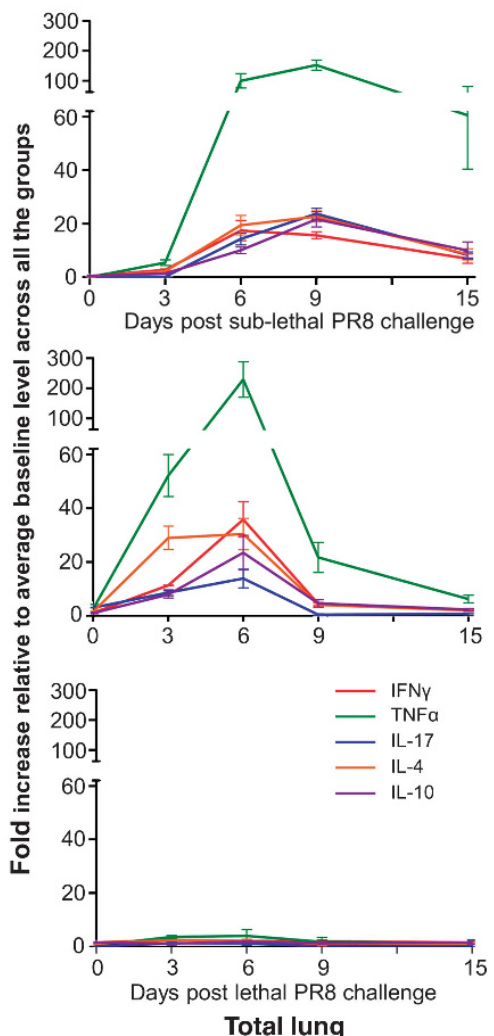

Figure 6 Airway and total lung CD8 $+\mathrm{NP}_{147-155}$ pentamer $+\mathrm{T}$-cell responses, viral loads, and T-cell cytokine burden after influenza challenge. Unimmunized mice $(n=22)$ were challenged with a sub-lethal $\left(0.8 \times L_{50}\right)$ dose of A/PR/8/34. vFLIP-NP (Kaposi's sarcoma-associated herpesvirus FLICE-like inhibitory protein-nucleoprotein) SC (subcutaneous; $n=20$ ) group mice were immunized twice subcutaneously with vFLIP-NP 50 ng 2 weeks apart, then challenged with $2 \times L_{50}$ of A/PR/8/34 2 weeks after the final immunization. vFLIP-NP SC-IN $(n=18)$ mice were immunized with vFLIP-NP $50 \mathrm{ng}$ subcutaneously followed by intranasal (IN) vFLIP-NP $200 \mathrm{ng} 2$ weeks later, then challenged with $2 \times \mathrm{LD}_{50}$ of A/PR/8/34 2 weeks after the final immunization. In all the groups, three mice were analyzed pre-challenge and on $3,6,9$, and 15 days after challenge. (a) $\mathrm{CD} 8+\mathrm{NP}{ }_{147-155}$ pentamer + T-cell numbers in $2 \times 2 \mathrm{ml}$ bronchoalveolar lavage per mouse. (b) CD8 $+\mathrm{NP}_{147-155}$ pentamer + numbers in total lung homogenate. Overlaid polygon indicates viral load by plaque assay adjusted for wet lung mass. (c) Levels of T-cell cytokines in lung homogenate (interferon $\gamma$ (IFN $\gamma$ ), tumor necrosis factor $\alpha(\mathrm{TNF} \alpha)$, interleukin-4 (IL-4), IL-10, IL-17), which have previously been implicated in lung damage during influenza infection. Levels are shown relative to the baseline (Day 0 ) average across all the three groups. Absolute values together with intracellular cytokine staining of CD8 + and CD4 $+\mathrm{T}$ cells are shown in Supplementary Figure S3. PFU, plaque-forming units.

phenotype $\left(\mathrm{CD}^{2} 2 \mathrm{~L}_{\mathrm{LO}}\right)$ antigen-specific $\mathrm{T}$ cells were present in the airway and lung. When these mice were infected with influenza, there was rapid and effective control of viral replication (Figure $\mathbf{6 b}$ ). The SC immunized group showed a later ( 3 days) and less effective (14-fold higher peak virus levels) viral clearance.

Both T-cell-derived IFN $\gamma$ and TNF $\alpha$ have a prominent role in lung injury. ${ }^{25,26}$ In humans, strong and early CD4 + Th17 responses have been associated with a more severe illness during the recent swine-origin H1N1 pandemic, and in knockout mouse models, IL-17 has been shown to be essential for lung injury, weight loss, and neutrophil infiltration but dispensable for viral clearance. ${ }^{27,28}$ Similarly, IL-4 and IL-10 $\mathrm{CD} 4+\mathrm{T}$-cell responses potentiate lung injury and airway hypersensitivity in influenza infection of mice without enhancing viral clearance or protection. ${ }^{28,29}$ We therefore examined total levels of these cytokines before and after influenza challenge in lung homogenate. In naive and SC immunized mice, numbers of pulmonary NP-specific T cells rose substantially 6 days after influenza infection, as did levels of TNF $\alpha$, IFN $\gamma$, IL-10, IL-4, and IL-17 (Figure $\mathbf{6 b}, \mathbf{c}$ ). The SC immunized group showed significantly greater $\mathrm{T}$-cell numbers and cytokine levels, despite higher peak viral titres in unimmunized mice, demonstrating the potential risks of this route of immunization. However, in lung homogenates from the SC-IN immunized mice, cytokines were minimal or absent (Figure $\mathbf{6 c}$; for absolute concentrations, see Supplementary Figure S3B).

\section{Antigen-specific CD8 + T-cell populations and protection are sustained for at least $\mathbf{4}$ months}

Generating long-lived protection against influenza is critical to effective vaccination coverage during an influenza season or emerging pandemic. We therefore investigated whether lung T-cell numbers and protection would be sustained 4 months after SC-IN vFLIP-NP immunization ("early recall" group). 
a
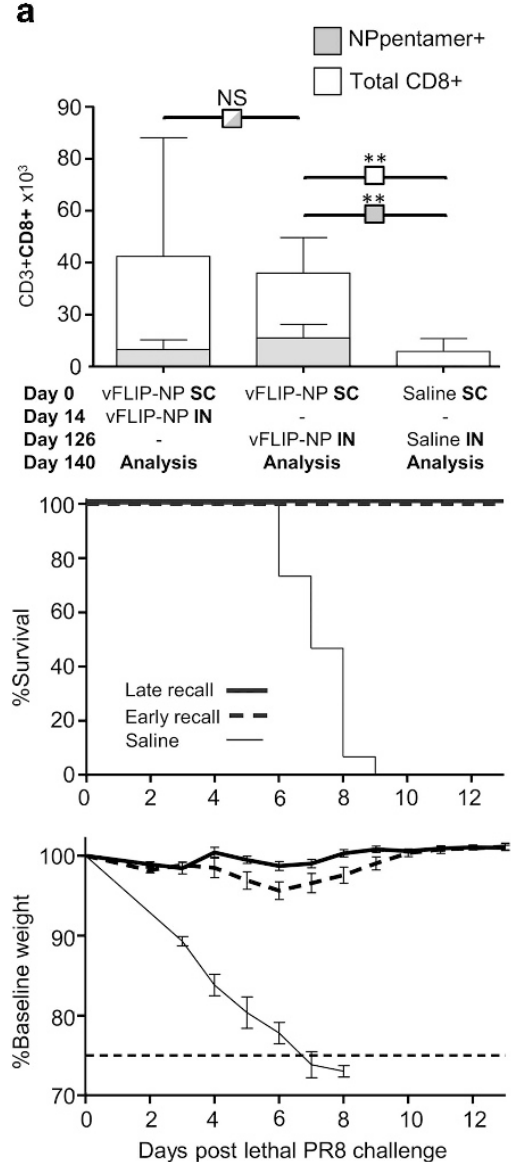

b
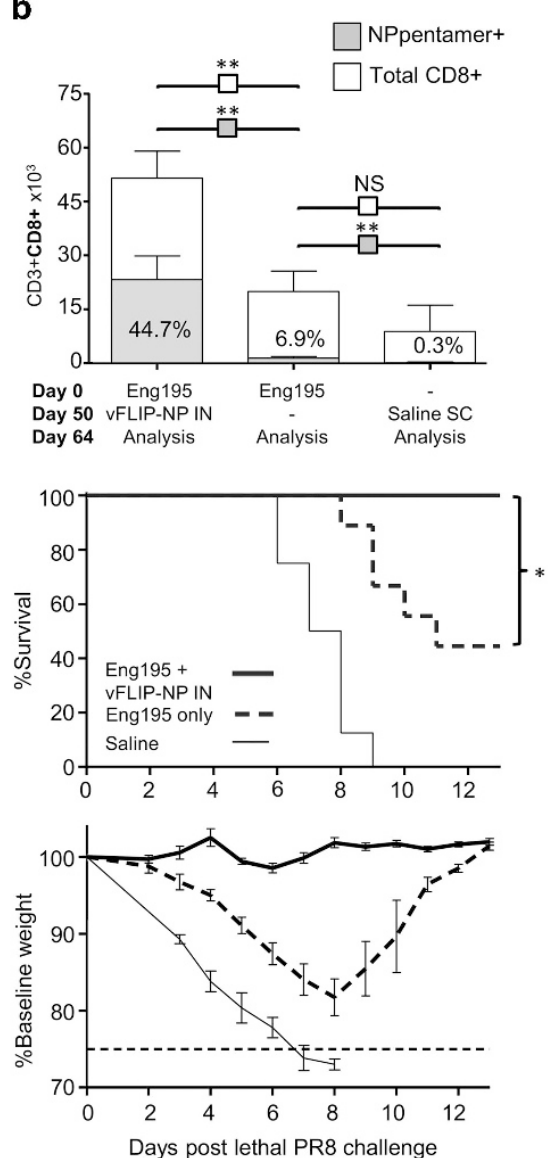

C

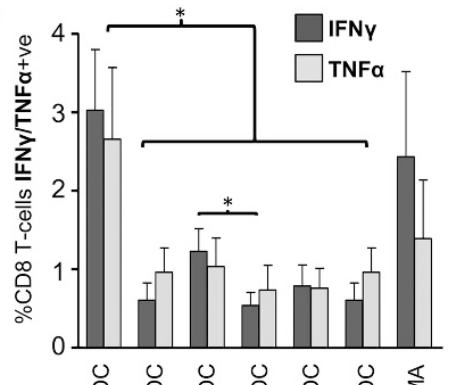

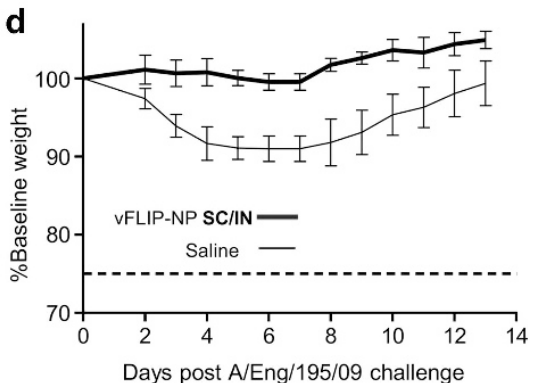

Figure 7 Longevity of SC-IN (subcutaneous followed by intranasal) vFLIP-NP (Kaposi's sarcoma-associated herpesvirus FLICE-like inhibitory proteinnucleoprotein) protection and application in clinically relevant models. (a) Long-term preservation of lung-based T-cell memory populations 4 months

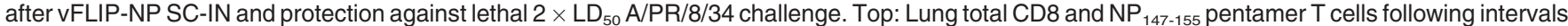
between subcutaneous and intranasal immunization and challenge indicated on the x axis. Levels were compared using Student's $t$-test. All mice were challenged on day 140 ( $n=10$ per group) or withdrawn for analysis ( $n=5$ per group). Survival and weight loss in survivors following challenge with lethal A/PR/8/24 are shown beneath. (b) Recall of naturally acquired T-cell memory to the lung by intranasal vFLIP-NP LV. Top: Lung total CD8 and NP $147-155$ pentamer CD8 + T cells 64 days after non-lethal challenge with A/Eng/195/09, with and without intranasal vFLIP-NP boosting 2 weeks before analysis ( $n=4$ per group). Percentages of total CD8 + T cells that are NP ${ }_{147-155}$ pentamer + are shown within bars. Survival and weight loss in survivors ( $n=12$ per group) are shown beneath following challenge with lethal A/PR/8/24 64 days after previous non-lethal infection with A/Eng/195/09. Mantel-Cox tests and Gehan-Breslow-Wilcoxon test produced similar $P$-values for observed differences in survival. (c) Human monocyte-derived dendritic cells (DCs) transduced with LV expressing NP recall autologous T-cell responses in vitro. peripheral blood mononuclear cells from HLA-A3-positive healthy volunteers were co-incubated with autologous DCs that had been transduced with LV (or matured with lipopolysaccharide (LPS) and interferon $\gamma$ (IFN $\gamma$ ) and pulsed with the HLA-A3-restricted peptide $\mathrm{NP}_{265-274}$ ). Cultures were re-stimulated with $\mathrm{NP}_{265-274}$ peptide before cytokine detection. To control for nonantigen-specific stimulation, DCs transduced with LV encoding hepatitis B core (HBc) were used. Gating strategy is shown in Supplementary Figure S4. Data shown are for five out of seven HLA-A3 subjects who responded to matured DC plus NP peptide. The percentage of positive CD8 T cells in each group were compared by the Wilcoxon's matched pairs test. (d) Cross-strain protection against A/Eng/195/09 conferred by SC-IN immunization with LV vFLIP-NP. Mice ( $n=10$ per group) were challenged with $5 \times 10^{4}$ plaque-forming units of A/Eng/195/09 2 weeks after the final immunization and weights monitored as previously. NS, not significant; PMA, phorbol myristate acetate.

Although a clinically applicable vaccine would require rapid induction of mucosal immunity and thus a short interval between SC and IN immunizations (as used above), the antigen-specific T-cell population 2 weeks after SC vaccination is likely a mix of waning primary effectors and established memory $\mathrm{T}$ cells. We therefore also examined whether IN immunization with LV 4 months after SC vaccination could recall a memory-only $\mathrm{T}$-cell population ("late recall" group).

Analysis of mouse lung homogenate 4 months after SC-IN immunization ("early recall", Figure 7a) revealed no significant difference compared with total lung CD8 + T-cell numbers in mice receiving vFLIP-NP IN 2 weeks before analysis (mean \pm s.e.m.: $9804 \pm 3288$ "early recall” vs. $16550 \pm 3920$ "late recall", $P=0.27$ ), with both the regimens conferring complete protection from lethal influenza challenge and preventing weight loss (Figure 7A). Numbers of $\mathrm{NP}_{147-155}$ pentamer positive CD8 + T cells extracted from lung 126 days after IN recall were also not significantly different from those seen 14 days after standard interval SC-IN vaccination with vFLIP-NP as used in other experiments (mean \pm s.e.m.: $9804 \pm 3288$ vs. $15810 \pm 1951, P=0.19)$. 


\section{Intranasal immunization can boost naturally acquired cross-strain immunity}

A SC immunization may be dispensable if naturally acquired T-cell memory can be boosted by IN vFLIP-NP immunization. A/Eng/195/09 infection resulted in $6.9 \% \mathrm{NP}_{147-155}$ pentamer + of total CD $8+\mathrm{T}$ cells in the lung 64 days later (Figure 7B), which conferred $44 \%$ protection against lethal $\mathrm{A} / \mathrm{PR} / 8 / 34$ challenge, though all survivors lost considerable weight, indicating the absence of effective cross-neutralizing antibodies against surface hemagglutinin or neuraminidase. However, vFLIP-NP IN boosting after non-lethal A/Eng/195/09 infection generated similar numbers of $\mathrm{NP}_{147-155}$ pentamer + positive $\mathrm{CD} 8+\mathrm{T}$ cells in the lungs as the SC-IN vFLIP-NP immunization (compare Figures $\mathbf{6 b}$ and $\mathbf{7 b}$ ). This vFLIPNP IN immunization of previously infected mice also conferred $100 \%$ protection without weight loss when mice were challenged with a lethal dose of A/PR/8/34 (Figure 7b).

vFLIP-NP recalls polyfunctional human CD8 + T-cell in vitro To test the principle that lentivirus-transduced antigenpresenting cells could recall human influenza T-cell responses, we transduced monocyte-derived DCs from HLA-A3 healthy volunteers (NP from A/PR/8/34 lacks a well-characterized HLA-A2 epitope), co-cultured the transduced DC with autologous lymphocytes, then measured expansion of IFN $\gamma$ or $\mathrm{TNF} \alpha$-secreting CD8 $+\mathrm{T}$ cells in vitro. Figure $7 \mathrm{C}$ shows that monocyte-derived human DCs transduced with vFLIP-NP LV stimulated significantly enhanced IFN $\gamma$ and TNF $\alpha$ CD $8+$ $\mathrm{T}$ cells compared with Null-NP transduced DCs or DCs matured with IFN $\gamma$ and LPS and pulsed with the HLAA3-restricted NP peptide. Use of LV expressing vFLIP with hepatitis $\mathrm{B}$ core $(\mathrm{HBc})$ did not induce T-cell responses, demonstrating that both vFLIP and NP are required (Figure $7 \mathrm{C}$ ).

\section{Intranasal immunization confers cross-strain protection against $\mathrm{H} 1 \mathrm{~N} 1$ pandemic influenza}

Although the presence of memory T cells specific to influenza NP have been repeatedly shown to cross-protect against other subtypes sharing the same dominant T-cell epitopes, a less examined question is whether this approach tolerates variation in NP sequence between strains. A/Eng/195/09 NP shares only 91\% amino-acid homology with A/PR/8/34 NP and while the $\mathrm{NP}_{147-155}$ class I epitope is preserved, the well-described $\mathrm{H} 2-\mathrm{K}^{\mathrm{d}}$ restricted CD4 epitope $\mathrm{NP}_{57-78}$ is non-identical. To determine whether the protection conferred by vFLIP-NP SC-IN immunization was maintained despite this sequence variation, mice were challenged with $5 \times 10^{4}$ plaque-forming units (PFU) of A/Eng/195/09 and monitored for weight loss. Whereas naive mice developed a prolonged illness losing approximately $10 \%$ of baseline weight, vFLIP-NP SC-IN immunized mice showed no signs of disease or weight loss (Figure $7 \mathbf{d}$ ).

\section{DISCUSSION}

The delicate architecture of the lung, adapted for gas exchange, is highly susceptible to disruption by inflammation. Homeostatic mechanisms thus exist to ensure a high threshold for inflammation initiation and its suppression after infection clearance. $^{30}$ This makes both priming and maintaining a protective T-cell population in the lung difficult. To date, a vaccination regimen that generates long-term mucosal T-cell protection against a highly pathogenic, mouse-adapted strain without clinical disease has not been demonstrated. Live attenuated influenza virus strains have been shown to generate protective $\mathrm{T}$-cell responses in mice against heterosubtypic influenza challenge, but protection is neither lasting nor without morbidity. ${ }^{31}$ A recent study using NP and matrix protein 2 (M2) in an adenoviral vector administered IN demonstrated lung NP-specific T-cell frequencies of $\sim 12 \%$ and protected all mice against a lethal dose of mouse-adapted A/FM/1/47 H1N1 but not without substantial weight loss. ${ }^{32}$

With IN administered LV, we have shown that it is possible to generate sufficiently large pulmonary T-cell populations to confer lasting protection against a highly pathogenic, mouse-adapted influenza infection without clinical disease. Importantly, we observed rapid viral elimination without accumulation of IFN $\gamma$, TNF $\alpha$, IL-17, IL-10, IL-4, or lung injury. Contrastingly, the T-cell response seen upon influenza infection of SC vFLIP-NP-immunized mice and also the response of naive mice to influenza infection are characterized by high total cytokine burdens. These levels are greatest when high $\mathrm{T}$ cell and viral loads coincide, but low in the absence of either component, as predicted by the T-cell adoptive intrapulmonary transfer experiments of Moskophidis and Kioussis, ${ }^{33}$ which anticipated that $\mathrm{T}$-cell vaccine strategies for influenza would have to generate an abundance of influenza-specific T-cell numbers in the lung very early in infection if T-cell-mediated injury is to be prevented.

The inability of IN LV immunization to prime T cells in the lung contrasts strikingly with its proficiency at recalling them. We propose that this is due to the distinct T-cell-priming abilities of the antigen-presenting cells transduced by these different routes of administration. DCs are largely absent from the airway space but abundant SC and are readily transduced by $\mathrm{LV}$ via this route. $^{34,35}$ Although DCs are sufficient and necessary for T-cell priming by SC LV, ${ }^{36} \mathrm{AM}$ are conventionally regarded as having a pivotal immunoregulatory role in the lung; ${ }^{22,37}$ under tonic suppression from signals from epithelium $\left(\mathrm{CD} 200,{ }^{38}\right.$ mucin-1, ${ }^{39}$ transforming growth factor- $\beta,{ }^{40}$ and surfactant ${ }^{41}$ ) while delivering regulatory signals or inadequate co-stimulation ${ }^{17}$ to $\mathrm{T}$ cells. However, vFLIP-mediated activation of $\mathrm{NF \kappa B}$ can subvert this role, generating significant T-cell chemokine secretion. T-cell memory-recall responses do not require strong co-stimulation but are highly sensitive to quantity and duration of antigen presentation. Both of these are maximized by the highly efficient transduction of large numbers of AM with a single IN administration of LV, which would account for the large $\mathrm{T}$-cell recall responses in the lung we have observed here. Chemokine secretion and antigen presentation by influenza-infected AM, on the other hand, may be short-lived as infection initiates apoptosis as well as NFKB activation leading to cell death within $20-30 \mathrm{~h}^{42}$ 
LV-transduced AM, by contrast, persist for the lifespan of the adult mouse. ${ }^{43}$

Although the quantity of mucosal CD8 $\mathrm{T}$ cells is clearly important for rapid viral control, we have also shown that $\mathrm{CD} 8+\mathrm{T}$ cells in the airway are significantly higher in Gzm B following recall with IN vFLIP-NP, compared with memory $\mathrm{T}$ cells generated in the spleen following SC priming, or airway $\mathrm{T}$ cells recalled by LV expressing antigen alone. Ex vivo human $\mathrm{T}$-cell recall cytokine responses against an NP epitope can also be enhanced by vFLIP activation of autologous antigenpresenting cells. SC-IN LV immunization would seem an ideal model with which to determine the precise co-signals from antigen-presenting cells that enhance mucosal memory T-cell function upon recall rather than priming-and how these may be manipulated for optimal therapeutic or prophylactic efficacy against respiratory pathogens and other lung diseases.

\section{METHODS}

Lentiviral production. LVs were derived from pHRSIN-CSGW as described previously. ${ }^{44}$ The ubiquitin (UBI) promoter was replaced with the phosphoglycerate kinase promoter for human DC experiments. CDNA for A/PR/8/34 NP was kindly provided by Dr Claire Bennett (UCL), HBc adw strain cDNA for antigen-specificity control was a gift from Dr Mike Whelan. Vesicular stomatitis virus G glycoprotein (VSV-G)-pseudotyped lentivectors were produced by cotransfection of the vector with pCMVR8.91 (Gag/pol) and pMDG (VSV-G envelope) as previously described. ${ }^{45}$ Supernatants were concentrated by ultracentrifugation at 24,000 r.p.m. through a $20 \%$ sucrose cushion. Titres were measured by reverse transcriptase assay (Roche Diagnostics, Burgess Hill, UK) and quantitative TaqMan PCR (Applied Biosystems, Warrington, UK). Expression of cloned inserts was confirmed by immunoblotting of transduced 293T cells using antiNP clone InA108 (Hytest, Turku, Finland), anti-vFLIP ${ }^{46}$ and anti-HBc clone 10e11 (Abcam, Cambridge, UK).

Analysis of AM and adoptive transfer. BAL was performed by instillation and retrieval of $2 \mathrm{ml}$ of HBSS (Hank's Balanced Salt Solution) through a 16F Venflon catheter (Becton Dickinson, Oxford, UK) into the transected trachea. Cells were centrifuged and re-suspended in $200 \mathrm{ul} \mathrm{FACS} \mathrm{buffer} \mathrm{for} \mathrm{staining} \mathrm{(after} \mathrm{blocking} \mathrm{with}$ anti-Fc receptor antibody) with anti-F4/80-PE (phycoerythrin) and anti-CD11c-APC (allophycocyanin; eBioscience, Hatfield, UK) before fixing and analysis by FACS. In adoptive transfer experiments, AMs were transduced in vivo by IN administration of LV at $200 \mathrm{ng}$ reverse transcriptase (RT) per mouse. Two days later, mice were killed and $2 \times 2 \mathrm{ml}$ BAL performed on each mouse. Cells were pooled within groups, washed, and re-suspended in HBSS and transferred IN into recipients. Two weeks after transfer, mice were either killed for analysis $(n=3)$ or challenged with lethal A/PR/8/24 influenza $(n=5)$.

Analysis of chemokine levels in BAL and cultured BAL supernatants. Levels of chemokines in a single $2 \mathrm{ml}$ BAL were measured by cytokine bead array (CBA) (FlowCytomix, eBioscience). Adherent cells from BAL were washed after overnight culture in RPMI 1640 5\% fetal calf serum and incubated for a further 4 days in $2 \mathrm{ml}$ fresh media without further washes. Supernatants were then collected for chemokine analysis and adherent cells removed with cell dissociation solution (Sigma, Poole, UK) for staining with anti-F4/80-PE and antiCD11c-APC and analysis as above.

Preparation and transduction of DC. Murine bone marrow-derived DCs were prepared as described previously ${ }^{47}$ and transduced on day 4 of culture in RPMI 1640 5\% fetal calf serum and $50 \mathrm{ng} \mathrm{ml}^{-1} \mathrm{GM}-\mathrm{CSF}$ (granulocyte macrophages colony-stimulating factor) at MOIs (multiplicities of infection) ensuring $>80 \%$ transduction. Control DC were matured with $100 \mathrm{ng} \mathrm{ml}^{-1}$ LPS. Three days after transduction, cells were harvested for staining and analysis by FACS. After Fcreceptor blockade, cells were stained with anti-CD11c-APC (eBiosciences) and either anti-mouse PE-conjugated intercellular adhesion molecule-1, CD40, PDL-1 (eBioscience), CD80, CD86, or MHC II (BD Biosciences, Oxford, UK). Cells were gated for analysis on $\mathrm{GFP}^{+} \mathrm{CD} 11 \mathrm{c}^{+}$for the transduced groups and CD $11 \mathrm{c}^{+}$for the control groups.

Immunization and influenza challenge. Female BALB/c mice, 6-8 weeks old, were purchased from Charles River Laboratories and kept in pathogen-free conditions. LV titration established a fall in CD8 T-cell responses measured by IFN $\gamma$ Elispot $<25 \mathrm{ng}$ RT. A dose of $50 \mathrm{ng} \mathrm{RT}$ was therefore given for SC immunization. Intranasal LV immunization was performed under ketamine and xylazine anesthesia by inoculation of $20 \mu \mathrm{l}$ of viral suspension into each nostril (total $200 \mathrm{ng}$ RT per mouse). This volume ensures aspiration into distal lung. Intranasal challenges with influenza virus were performed in the same way. Following challenge, mice were weighed daily from Day 3 onwards and clinical signs of disease were scored. Mice were killed if weight loss exceeded $25 \%$.

Influenza strains used were mouse-adapted A/PR/8/34 (a kind gift from Dr Mike Whelan, UCL) and A/Eng/195/09, an $\mathrm{H} 1 \mathrm{~N} 1$ strain from the recent swine-origin H1N1 pandemic. Viruses were titrated by plaque assay in MDCK cell layers inoculated with $100 \mu \mathrm{l}$ serially diluted samples and incubated for 3 days after overlay with $0.6 \%$ agarose (oxoid) in Dulbecco's modified Eagle's medium with $2 \mu \mathrm{g} \mathrm{ml}^{-1}$ trypsin (Worthington). A dose of $2 \times \mathrm{LD}_{50}$ corresponded to 2,500 PFU per mouse by plaque assay. This gave $100 \%$ lethality in all control mice $(n>70)$. Where specified as "sub-lethal" challenges a 1,000 PFU dose was given to unimmunized mice. A/Eng/ $195 / 09$ is non-lethal in mice and was used at $5 \times 10^{4} \mathrm{PFU}$ per mouse.

Immunoanalysis. For quantitative comparisons of airway $\mathrm{T}$ cells between mice, a single $2 \mathrm{ml} \mathrm{BAL}$ was performed. Lungs were dissected and mediastinal lymph nodes trimmed away. After weighing, lungs were mashed through a $70 \mu \mathrm{m}$ sieve and leukocytes isolated by separation over a histopaque (Sigma) density gradient.

Cells isolated from airway, lung, spleen, or circulation were either stained immediately to quantify and phenotype NP-specific T cells with one of the two panels $\left(\mathrm{NP}_{147-155}\right.$ pentamer-PE (ProImmune, Oxford, UK), anti-CD8-APC, CD19-FITC, CD62L, and CD127eFluor 450 (eBioscience) or $\mathrm{NP}_{147-155}$ pentamer-PE, live/dead-APCcy7, anti-CD3-PERCPcy5, anti-CD4-v500, anti-CD8-AF488, anti-GzmBAPC, anti-Ki67-FITC) or alternatively stimulated overnight with relevant peptide and brefeldin $\mathrm{A}\left(1 \mu \mathrm{g} \mathrm{ml}^{-1}\right)$ in the last $5 \mathrm{~h}$ before staining (live/dead-APCcy7, anti-CD3-PERCPcy5, anti-CD4-v500, anti-CD8-v450) followed by permeabilization for intracellular cytokine staining (anti-IL-2-PeCy7, anti-GzmB-APC, anti-IFN $\gamma$-FITC, anti-TNF $\alpha$-PE). For re-stimulation of $\mathrm{CD} 8+\mathrm{T}$-cell responses in culture, the $\mathrm{NP}_{147-155}$ TYQRTRALV peptide, a H2- $\mathrm{K}^{\mathrm{d}}$-restricted CD8 epitope $^{48}$ was used. For re-stimulation of CD4 T-cell responses, the peptide $\mathrm{NP}_{55-78}$ RLIQNSLTIERMVLSAFDERRNKY was used. ${ }^{49}$ In addition to intracellular cytokine staining analysis of lung CD4 T cells, splenocytes were re-stimulated for 4 days in the presence or absence of peptide $\mathrm{NP}_{55-78}$ in RPMI 5\% fetal calf serum. Supernatants were then harvested and frozen for cytokine analysis by cytometric bead array (TH-1/TH-2 FlowCytomix kit, eBiosciences).

Human influenza immune responses. Seven HLA-A3-positive adult healthy volunteers (all aged $>25$ years) were identified by screening with anti-HLA3 antibody staining of peripheral blood mononuclear cells (PBMCs). Sixty milliliters of peripheral blood was collected and PBMC isolated by Ficoll-Hypaque density centrifugation separation. Monocytes were isolated from PBMC by positive anti-CD14 magnetic bead separation through LS columns (Miltenyi Biotech, Bisley, UK). 
The CD14-negative PBMC fraction was frozen and CD14-positive cells cultured in X-vivo media (BioWhittaker, Wokingham, UK) supplemented with $1 \%$ human AB serum and GM-CSF and IL-4 (both at $50 \mathrm{ng} \mathrm{ml}^{-1}$ ) for 4 days. Non-adherent cells were harvested and transduced with LV at an MOI of 60 in the presence of $10 \mu \mathrm{g} \mathrm{ml}^{-1}$

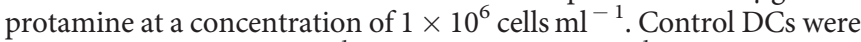
matured with $100 \mathrm{IU} \mathrm{ml}^{-1}$ IFN $\gamma$ and $100 \mathrm{ng} \mathrm{ml}^{-1}$ LPS and pulsed with either HLA-matched $\mathrm{NP}_{265-274}$ class I peptide (ILRGSVAHK) or irrelevant $\mathrm{HBc}_{18-27}$ peptide. Cells were incubated for 3 days in 96-well plates before washing and re-suspending in media with addition of CD14-depleted autologous PBMCs for 10 days, replenishing $50 \%$ of the media at day 5. Before FACS analysis, cells were stimulated with the ILRGSVAHK peptide overnight in the presence of $1 \mathrm{ug} \mathrm{ml}^{-1}$ brefeldin A. PBMC were stained for live/dead (Invitrogen), anti-human CD3 -PE-Cy7, CD4-APC-Cy7, CD8 -AF700 (eBiosciences), and permeabilized for intracellular staining of IFN $\gamma$-APC, IL-2-FITC, and TNF $\alpha$-PE (BD Biosciences). Cells were acquired on a BD LSR II and analyzed with Flowjo software (Tree Star, OR, USA). Non-response was defined as no increase in IFN $\gamma$ positive CD8 T-cell populations in cultures stimulated with LPS/IFN $\gamma$-matured DC pulsed with ILRGSVAHK peptide compared with irrelevant peptide. Five of the seven HLA-A3 subjects responded and were used for further analysis.

Statistical analyses. All data were analyzed using the GraphPad Prism v5.0 statistical software package (GraphPad Software, Inc., La Jolla, CA). Statistical tests applied to each data set are indicated in the relevant figure legend.

SUPPLEMENTARY MATERIAL is linked to the online version of the paper at http://www.nature.com/mi

\section{ACKNOWLEDGEMENTS}

This work was funded by a Medical Research Council Clinical Research Training Fellowship to D.C.M. We are grateful to Professor Sarah Gilbert and Professor Adrian Hill for their useful advice throughout this project.

\section{DISCLOSURE}

The authors declared no conflict of interest.

c) 2014 Society for Mucosal Immunology

\section{REFERENCES}

1. Zweerink, H.J., Courtneidge, S.A., Skehel, J.J., Crumpton, M.J. \& Askonas, B.A. Cytotoxic T cells kill influenza virus infected cells but do not distinguish between serologically distinct type A viruses. Nature 267, 354-356 (1977).

2. Yap, K.L., Ada, G.L. \& McKenzie, I.F. Transfer of specific cytotoxic $T$ lymphocytes protects mice inoculated with influenza virus. Nature $\mathbf{2 7 3}$, 238-239 (1978).

3. Liang, S., Mozdzanowska, K., Palladino, G. \& Gerhard, W. Heterosubtypic immunity to influenza type A virus in mice. Effector mechanisms and their longevity. J. Immunol. 152, 1653-1661 (1994).

4. Webster, R.G. \& Askonas, B.A. Cross-protection and cross-reactive cytotoxic $T$ cells induced by influenza virus vaccines in mice. Eur. J. Immunol. 10, 396-401 (1980).

5. Donnelly, J.J. et al. Preclinical efficacy of a prototype DNA vaccine: enhanced protection against antigenic drift in influenza virus. Nat. Med. 1, 583-587 (1995)

6. McMichael, A.J., Gotch, F., Cullen, P., Askonas, B. \& Webster, R.G. The human cytotoxic $T$ cell response to influenza $A$ vaccination. Clin. Exp. Immunol. 43, 276-284 (1981).

7. Berkhoff, E.G.M., Geelhoed-Mieras, M.M., Fouchier, R.A.M., Osterhaus, A.D.M.E. \& Rimmelzwaan, G.F. Assessment of the extent of variation in influenza A virus cytotoxic T-lymphocyte epitopes by using virus-specific CD8 + T-cell clones. J. Gen. Virol. 88, 530-535 (2007).

8. Lillie, P.J. et al. Preliminary assessment of the efficacy of a T-cell-based influenza vaccine, MVA-NP + M1, in humans. Clin. Infect. Dis. 55, 19-25 (2012).
9. Flynn, K.J. et al. Virus-specific CD8 + T cells in primary and secondary influenza pneumonia. Immunity 8, 683-691 (1998).

10. Ely, K.H., Cookenham, T., Roberts, A.D. \& Woodland, D.L. Memory T cell populations in the lung airways are maintained by continual recruitment. J. Immunol. 176, 537-543 (2006).

11. Nguyen, H.H. et al. Heterosubtypic immunity to lethal influenza A virus infection is associated with virus-specific CD8 + cytotoxic Tlymphocyte responses induced in mucosa-associated tissues. Virology 254, 50-60 (1999),

12. Lund, F.E. et al. Lymphotoxin-alpha-deficient mice make delayed, but effective, Tand B cell responses to influenza. J. Immunol. 169, 5236-5243 (2002).

13. Mrusek, S., Vallbracht, S. \& Ehl, S. The impact of splenectomy on antiviral T cell memory in mice. Int. Immunol. 17, 27-33 (2005).

14. Neutra, M.R. \& Kozlowski, P.A. Mucosal vaccines: the promise and the challenge. Nat. Rev. Immunol. 6, 148-158 (2006).

15. Naldini, L. et al. In vivo gene delivery and stable transduction of nondividing cells by a lentiviral vector. Science 272, 263-267 (1996).

16. Buckley, S.M.K. et al. Lentiviral transduction of the murine lung provides efficient pseudotype and developmental stage-dependent cell-specific transgene expression. Gene Ther. 15, 1167-1175 (2008).

17. Chelen, C.J. et al. Human alveolar macrophages present antigen ineffectively due to defective expression of B7 costimulatory cell surface molecules. J. Clin. Invest. 95, 1415-1421 (1995).

18. Rowe, H.M. et al. Expression of vFLIP in a lentiviral vaccine vector activates NF-\{kappa\}B, matures dendritic cells, and increases CD8 + T-cell responses. J. Virol. 83, 1555-1562 (2009).

19. Shimizu, A. et al. Kaposi's sarcoma-associated herpesvirus vFLIP and human $T$ cell lymphotropic virus type 1 Tax oncogenic proteins activate IkappaB kinase subunit gamma by different mechanisms independent of the physiological cytokine-induced pathways. J. Virol. 85, 7444-7448 (2011).

20. Sprenger, $\mathrm{H}$. et al. Selective induction of monocyte and not neutrophilattracting chemokines after influenza A virus infection. J. Exp. Med. 184, 1191-1196 (1996).

21. Kaufmann, A. et al. Defense against influenza A virus infection: essential role of the chemokine system. Immunobiology 204, 603-613 (2001).

22. Thepen, T., Van Rooijen, N. \& Kraal, G. Alveolar macrophage elimination in vivo is associated with an increase in pulmonary immune response in mice. J. Exp. Med. 170, 499-509 (1989).

23. Van Rijt, L.S. et al. In vivo depletion of lung CD11C + dendritic cells during allergen challenge abrogates the characteristic features of asthma. J. Exp. Med. 201, 981-991 (2005).

24. Crowell, R.E., Heaphy, E., Valdez, Y.E., Mold, C. \& Lehnert, B.E. Alveolar and interstitial macrophage populations in the murine lung. Exp. Lung Res. 18, 435-446 (1992).

25. Graham, M.B. et al. Response to influenza infection in mice with a targeted disruption in the interferon gamma gene. J. Exp. Med. 178, 1725-1732 (1993).

26. Hussell, T., Pennycook, A. \& Openshaw, P.J. Inhibition of tumor necrosis factor reduces the severity of virus-specific lung immunopathology. Eur. J. Immunol. 31, 2566-2573 (2001).

27. Bermejo-Martin, J.F. et al. Th1 and Th17 hypercytokinemia as early host response signature in severe pandemic influenza. Crit. Care 13, R201 (2009).

28. Crowe, C.R. et al. Critical role of IL-17RA in immunopathology of influenza infection. J. Immunol. 183, 5301-5310 (2009).

29. Graham, M.B. \& Braciale, T.J. Resistance to and recovery from lethal influenza virus infection in B lymphocyte-deficient mice. J. Exp. Med. 186, 2063-2068 (1997).

30. Snelgrove, R.J., Godlee, A. \& Hussell, T. Airway immune homeostasis and implications for influenza-induced inflammation. Trends Immunol. 32, 328334 (2011)

31. Powell, T.J. et al. Priming with cold-adapted influenza A does not prevent infection but elicits long-lived protection against supralethal challenge with heterosubtypic virus. J. Immunol. 178, 1030-1038 (2007).

32. Price, G.E. et al. Single-dose mucosal immunization with a candidate universal influenza vaccine provides rapid protection from virulent $\mathrm{H} 5 \mathrm{~N} 1$, H3N2 and H1N1 viruses. PLoS One 5, e13162 (2010).

33. Moskophidis, D. \& Kioussis, D. Contribution of virus-specific CD8+ cytotoxic Tcells to virus clearance or pathologic manifestations of influenza 


\section{ARTICLES}

virus infection in a T cell receptor transgenic mouse model. J. Exp. Med. 188, 223-232 (1998).

34. He, Y., Zhang, J., Donahue, C. \& Falo, L.D. Jr Skin-derived dendritic cells induce potent CD $8(+)$ Tcell immunity in recombinant lentivector-mediated genetic immunization. Immunity 24, 643-656 (2006).

35. Rowe, H.M. et al. Immunization with a lentiviral vector stimulates both CD4 and CD8 T cell responses to an ovalbumin transgene. Mol. Ther. 13, 310-319 (2006).

36. Goold, H.D., Escors, D., Conlan, T.J., Chakraverty, R. \& Bennett, C.L. Conventional dendritic cells are required for the activation of helperdependent CD8 T cell responses to a model antigen after cutaneous vaccination with lentiviral vectors. J. Immunol. 186, 4565-4572 (2011).

37. Holt, P.G. Inhibitory activity of unstimulated alveolar macrophages on T-lymphocyte blastogenic response. Am. Rev. Respir. Dis. 118, 791-793 (1978).

38. Snelgrove, R.J. et al. A critical function for CD200 in lung immune homeostasis and the severity of influenza infection. Nat. Immunol. 9, 1074-1083 (2008).

39. Ueno, K. et al. MUC1 mucin is a negative regulator of Toll-like receptor signaling. Am. J. Respir. Cell Mol. Biol. 38, 263-268 (2008).

40. Munger, J.S. et al. The integrin alpha $v$ beta 6 binds and activates latent TGF beta 1: a mechanism for regulating pulmonary inflammation and fibrosis. Cell 96, 319-328 (1999).

41. Haczku, A. Protective role of the lung collectins surfactant protein $A$ and surfactant protein D in airway inflammation. J. Allergy Clin. Immunol. 122, 861-879. quiz 880-881 (2008).
42. Fesq, H., Bacher, M., Nain, M. \& Gemsa, D. Programmed cell death (apoptosis) in human monocytes infected by influenza A virus. Immunobiology 190, 175-182 (1994).

43. Wilson, A.A. et al. Amelioration of emphysema in mice through lentiviral transduction of long-lived pulmonary alveolar macrophages. J. Clin. Invest. 120, 379-389 (2010).

44. Demaison, C. et al. High-level transduction and gene expression in hematopoietic repopulating cells using a human immunodeficiency [correction of imunodeficiency] virus type 1-based lentiviral vector containing an internal spleen focus forming virus promoter. Hum. Gene Ther. 13, 803-813 (2002).

45. Besnier, C., Takeuchi, Y. \& Towers, G. Restriction of lentivirus in monkeys. Proc. Natl. Acad. Sci USA 99, 11920-11925 (2002).

46. Low, W. et al. Internal ribosome entry site regulates translation of Kaposi's sarcoma-associated herpesvirus FLICE inhibitory protein. J. Virol. 75, 2938-2945 (2001).

47. Inaba, K. et al. Granulocytes, macrophages, and dendritic cells arise from a common major histocompatibility complex class II-negative progenitor in mouse bone marrow. Proc. Natl. Acad. Sci. USA 90, 3038-3042 (1993).

48. Bodmer, H.C., Pemberton, R.M., Rothbard, J.B. \& Askonas, B.A. Enhanced recognition of a modified peptide antigen by cytotoxic T cells specific for influenza nucleoprotein. Cell 52, 253-258 (1988).

49. Gao, X.M., Liew, F.Y. \& Tite, J.P. Identification and characterization of T helper epitopes in the nucleoprotein of influenza A virus. J. Immunol. 143, 3007-3014 (1989). 